\title{
Systematic Analysis of Clinical Efficacy of Acupuncture on Treating Pediatric Cerebral Palsy Dyskinesia
}

\author{
Yujin Hou, Xiaosu Jie, Xin Guo, Yingying Zhang, Hua Shi, Yuanjun Lou
}

Henan Province Hospital of Traditional Chinese Medicine, Henan, Zhengzhou, 450002

Keywords: Pediatric cerebral palsy dyskinesia, Clinical Efficacy, Systematic Analysis

\begin{abstract}
Pediatric cerebral palsy refers to non-progressive brain injury syndrome with central motor dysfunction and postural abnormalities as the main manifestation of various brain pathologies during the period from birth before birth to 1 month after birth accompanied by varying degrees of mental and hearing loss, mental retardation, epilepsy, speech impairment, visual, behavioral and sensory abnormalities and other symptoms. The incidence of pediatric cerebral palsy in China is $1 \%-5 \%$ [1], which affected the physical and mental health of the sick children. At present, children with cerebral palsy is still lack of effective treatment drugs, there is a variety of rehabilitation therapies, while acupuncture in traditional Chinese medicine in the filed plays an important role in the treatment. In this study, from January 2010 to October 2012, 72 cases of children with cerebral palsy admitted to Henan Province Hospital of Traditional Chinese Medicine were observed, and the effect of acupuncture and moxibustion combined with exercise training on children with cerebral palsy was analyzed.
\end{abstract}

\section{Introduction}

Cerebral palsy refers to non-progressive brain injury syndrome caused by various causative factors in the process of brain development from birth to birth within 1 month, which is mainly characterized by central movement disorders and postural abnormalities, and is often accompanied by different Degree of mental retardation, mental retardation, epilepsy, language and visual, auditory, behavioral and cognitive disorders and other obstacles, seriously affecting children's physical and mental health. The incidence of pediatric cerebral palsy at home and abroad were 1 . $8 \% \sim 4 \%$, $1 \%$ \% 5 \% [1]. Cerebral palsy is currently lack of treatment for specific drugs, therefore, the mainstream of treatment at home and abroad is mainly based on the treatment of rehabilitation, traditional medicine acupuncture also plays an important role in rehabilitation. The author of 85 cases of children with cerebral palsy treated in our department from May 2008 to October 2010 were observed, of which 43 cases were given electroacupuncture mainly combined with exercise training and the use of coarse motor function test table before and after treatment to change the dynamic function of motor function Evaluation method.

\section{Materials and Methods}

From May 2013 to October 2015, 85 children with cerebral palsy treated by rehabilitation department in our hospital. There were 45 males and 40 females, aged from 6 months to 6 years old. All children diagnosed in line with 2006 National Cerebral Palsy Symposium standards and classification [2], have motor dysfunction, excluding children with secondary epilepsy. Eighty-five children were numbered according to the order of their visit, and a table was prepared of the GMFM score for each child prior to treatment for gross motor function. A single number of 43 children were taken as treatment group and acupuncture plus motor training. 42 cases of double-control group, only simple exercise function training. Two groups of gender, age, clinical type no significant difference $(\mathrm{P}>0.05)$.

(1) meet the diagnostic criteria of cerebral palsy; (2) age of 6 months to 6 years of age; (3) no obvious heart and lung organic disease, no secondary epilepsy; no fever, the needle site without skin damage and skin infections, no spontaneous bleeding Tendency and other blood diseases, etc.; (4) to 
comply with the requirements of the therapist and insist on treatment. The treatment group used acupuncture combined with motor function training. The control group only used motor function training.

According to the condition of children, acupoints are selected: (1) Scalp acupuncture: Sishencong (head and Baihui center and 1 inch each) The first pin, before and after the next 1-inch for the 2,3-pin), top anterior oblique line, the top center line, the top next to a line, the top 2 lines. Spasms do not use strong stimulation; Xu move without needle. (2) body acupuncture plus upper limb paralysis plus pool, Hegu, Shousanli, shoulder Yu; lower limb paralysis plus Zusanli, Xie River, Sanyinjiao, Bi off, ring jump, V rabbit. Operation: Each time taking the above acupuncture points 7 to 8, conventional disinfection, left index finger or thumb tight according to the acupuncture point, with Huapo brand $0.3 \mathrm{~mm} \times 25 \mathrm{~mm}$ acupuncture needles, needle inserted quickly twist twisting, after gas Connect the SDZ - II electroacupuncture instrument to determine the intensity knob zero, turn off the power, the use of density of the wave, the output of each pair of electro-acupuncture were connected to the same innervation of muscle two acupoints, the power adjustment strength Knob, gradually increased to mild muscle contraction, children can tolerate the degree. Continuous power 30 min, 1 day, 10 times a course of treatment. Intermission for 10 days to continue the next course of treatment. Continuous treatment for 6 months.

Specific types of children with cerebral palsy diagnosed and recent problems identified, according to the disease to develop rehabilitation programs, short-term and long-term goals, therapists according to different abnormalities in children for 1 month of rehabilitation training, the use of physical therapy (PT) to reduce or stabilize the muscle tension, and then to Bobath inhibition of abnormal posture, Vojta method to promote normal exercise, according to the law of child growth and development, from the head-turn-sit-kneeling- climb- station- walk training, according to different The condition of the head control and upper limbs, trunk and other balance training. Repeatedly strengthen, guide the completion of the action, 1 times a day, every $30 \min \sim 1 \mathrm{~h}$. Combination of homework training exercises upper and lower extremity function and the fine movement of the hand treatment, 10 days a course of treatment, 10 days after the rest of the treatment. This cycle of 6 months. The recovery of limb motor function before and after treatment was assessed by the gross motor function assessment scale (GM-FM) [3]. The scale includes 88 items, divided into lying and stand up, sitting and kneeling, standing position and walking and running jump five functional areas. Each of these functional areas of each indicator according to the degree of completion of children score, can not be 0 points; to complete the action $<10 \%$ for 1 point; $10 \%$ to $100 \%$ for 2 points; 3 points for all completed. Two groups of children before treatment and 6 months after treatment each made a rating, observe the recovery of motor function. National pediatric cerebral palsy academic seminar summary standard [4]. Markedly: exercise development, joint activity, coordination function, the original reflex and automatic response and muscle strength and other items are close to normal children of the same age; effective: physical activity, joint activity, coordination, the original reflex and automatic response and muscle strength Items were significantly improved compared with before treatment; invalid: no change in the above indicators before and after treatment.

\section{Statistical analysis}

After 6 months of treatment, children with cerebral palsy improved motor function in both groups. Compared with the control group, the total effective rate $(90.7 \%$ vs. $66.7 \%)$ was statistically significant $(\mathrm{P}<0.01)$. Before and after treatment, the two groups of patients with gross motor function GMFM score control results in Table 3,43 treatment group patients before treatment, the mean gross motor function GMFM score was $104.3 \pm 53.8$, after treatment, gross motor function GMFM score was 168. $5 \pm 49.1 .42$ cases of control group before treatment, the average gross motor function GMFM score was $108.5 \pm 52$. 3, after treatment, the gross motor function GMFM score was $134.1 \pm 46$. 5 . The two groups of patients before treatment, gross motor function GMFM score control showed no significant difference $\mathrm{P}>0.05$. After treatment scores were significantly different, the difference was statistically significant $(\mathrm{P}<0.01)$. From Table 4 , the efficacy of patients 
aged 6 months to 3 years was significantly better than that of patients aged 3 to 6 years $(\chi 2=7.45$, $\mathrm{P}<0.01$ ), indicating that the younger the effect is, the better.

\section{Discussion}

The main features of cerebral palsy manifested as abnormal movement and posture. To spastic more common, due to children with high muscle tension, abnormal posture and joint stiffness is relatively rigid, limiting the ability of children's daily living activities. Children with cerebral palsy diagnosed, they need to be treated. Comprehensive rehabilitation is the main method of treatment of cerebral palsy at home and abroad. The research on traditional medical treatment in China is increasing year by year. Acupuncture in traditional medicine also plays an important role in rehabilitation. Comprehensive treatment of Integrative Medicine and Western rehabilitation measures, and achieved a positive effect, greatly improving the quality of life of children with cerebral palsy. Cerebral palsy motor dysfunction, postural abnormalities in the motherland medicine belongs to "five late", "five soft", "atrophy syndrome" and other fields. The pathogenesis of congenital endowment deficiency or acquired dystrophy, so that liver and kidney spleen or blood stasis resistance, lesions in the head. The head of Zhu Yang will be an important part of the gathering of qi and blood. Scalp acupuncture treatment is an important means of medical treatment of acupuncture in the motherland, acupuncture and moxibustion based on the theory of horizontal contact, combined with modern medical anatomy and physiology summarized. The acupuncture site is located in the cerebral cortex corresponding to the scalp projection area, according to different conditions of cerebral palsy children with brain injury acupuncture. Known scalp acupuncture expert Lin Xuejian that the brain is a network of nerve cells [5]. The principle of scalp acupuncture is to improve the blood supply of neurons in local area, promote the functional metabolism of brain cells, rebuild the network of neurons, promote the recovery of motor function in children, and treat brain-derived diseases. According to the degree of limb dysfunction in children, plus local acupoints, play to adjust the function of organs, balance yin and yang, conditioning meridian qi and blood. The points echo each other, to enhance the stimulation of electro-acupuncture, prompting systemic qi and blood Meridian through. Motor function training is aimed at the abnormal posture left behind by cerebral palsy and a variety of movement disorders, and a series of exercise therapy, prolonged repeated stimulation to relieve symptoms to improve motor function of a method. It is a recognized international and domestic feasible method of treatment of cerebral palsy. In the author's research, with the training of modern rehabilitation motor function at the same time, the main acupuncture treatment, as a comprehensive rehabilitation of cerebral palsy. The results show that modern rehabilitation exercise training and acupuncture coordination, can play a coordinating role. In 43 cases of exercise therapy plus acupuncture, 25 cases were markedly effective, 14 cases were effective, the total effective rate was $90.7 \%$, compared with the control group, the treatment effect was significant. Shi Bingpei that the GMFM scale is specifically for children with cerebral palsy coarse motor assessment method, and Gesell scale and pediatric neuropsychological development checklist compared to more accurately reflect the subtle changes in gross motor development, better assessment of motor function Improve the situation. In this study, the gross motor function scale was used to evaluate and quantify. There was no significant difference between the two groups in GMFM scores before treatment, but there was significant difference between the two groups after treatment. The study found that with the improvement of clinical symptoms, GMFM score is also rising, more objectively reflect the improvement of motor function. In summary, I believe that acupuncture as a traditional Chinese treatment can effectively improve the treatment of children with cerebral palsy, its motor function improved significantly. Acupuncture combined with motor function training for children with cerebral palsy is better than simple exercise therapy. In the study, 85 children with cerebral palsy were treated for statistical analysis and found that the clinical efficacy of patients aged 6 months to 3 years was significantly better than 3 to 6 years old, indicating that the younger, the greater the plasticity of the brain, the better the effect. Therefore, early diagnosis and treatment of early intervention is the key to cerebral palsy. Cerebral palsy rehabilitation in children is a complex and multidisciplinary process. As a kind of holistic 
rehabilitation method, acupuncture has unique advantages. Acupuncture and Rehabilitation as a non-toxic side effect of green therapy, easy to operate, easy to promote. Therefore, promoting the application of acupuncture and moxibustion in the rehabilitation of cerebral palsy, further improving and standardizing the operation of traditional rehabilitation and gradually forming a unified treatment plan will be the focus and direction for future work.

\section{Conclusions}

By comparing two groups of patients before treatment, gross motor function measurement scale (GMFM) score analysis, the difference was not significant, the control after treatment showed that the difference was significant. Therefore, acupuncture can effectively improve the treatment of children with cerebral palsy, significantly improve their motor function.

\section{References}

[1] Wang Xiaochen, Chen Yi, Sun Zhiying, et al. Acupuncture treatment of children with cerebral palsy hearing impairment clinical observation [J]. Journal of Acupuncture and Moxibustion, 2011, 27 (7): 19 - 21

[2] Hu Yingyuan. Rehabilitation management of children with cerebral palsy [J]. Chinese Rehabilitation Theory and Practice, 2006,12 (2): 112 - 113

[3] Liu Peng, Huang Dongfeng, Jiang Qin. Standardized study of measuring scale of gross motor function in children with cerebral palsy [J]. Chinese Journal of Rehabilitation Medicine, 2004,19 (3): $170-173$

[4] Deng Jin'e, Pan Qiulan, Zhang Limei. Scalp acupuncture combined with modern rehabilitation of children with cerebral palsy on motor function and mental development [J]. Chinese Journal of Clinical Rehabilitation, 2005,9 (7): 120-121

[5] Wang Haili, Wu Jiwei, Lin Xuejian. Lin Xuejian use scalp acupuncture treatment of brain disease experience [J]. China Acupuncture, 2005,25 (10): 729 - 732 